EPJ Web of Conferences 41, 05007 (2013)

DOI: $10.1051 /$ epjconf/20134105007

(C) Owned by the authors, published by EDP Sciences, 2013

\title{
Ultrafast CARS with Improved Spectral Resolution
}

\author{
M. Lütgens, S. Chatzipapadopoulos, and S. Lochbrunner \\ Institut für Physik, Universität Rostock, Universitätsplatz 3, 18051 Rostock, Germany
}

\begin{abstract}
Molecular vibrations are investigated by time and frequency resolved CARS applying ultrafast excitation and picosecond probing for high spectral resolution. Enhanced spectral structure and beating phenomena are demonstrated for coalescing Raman bands.
\end{abstract}

\section{Introduction}

Coherent anti-Stokes Raman scattering (CARS) is a powerful method for the spectroscopy of vibrations. Using a combination of femto- and picosecond light sources for broadband excitation and narrowband probing allows monitoring the temporal evolution of a whole set of excited vibrational coherences with high spectral resolution. In liquids the line widths of vibrational modes measured by linear Raman are broadened by interactions with the environment causing accelerated dephasing. In contrast to linear Raman, CARS is based on probing excited coherences. Individual molecular modes which do not vibrate in phase with the ensemble average are not contributing to the measured signal field [1]. Consequently homogeneous dephasing reduces the signal but does not spectrally broaden the CARS lines. Applying narrowband Raman probe pulses in CARS leads therefore to highly resolved spectra and may reveal signatures hidden in broad bands consisting of overlapping vibrational transitions [1].

We present a new setup based on two non-collinear optical parametric amplifiers (NOPAs) to record such CARS spectra and discuss first experimental results for closely spaced transitions in cyclohexane and overlapping $\mathrm{CH}$-stretch vibrations of an ionic liquid. Beating phenomena and an increase of spectral structure are shown.

\section{CARS setup and signal analysis}

The interacting laser fields are generated by a modified NOPA which delivers narrowband picosecond pulses for the CARS pump and probe beam and a conventional femtosecond NOPA providing ultrashort Stokes pulses. Both are pumped by a Ti:sapphire amplifier system. The modified NOPA consists of a single stage NOPA, a grating monochromator, which filters spectrally the output of the first stage, and a second parametric stage, which amplifies the output of the monochromater and which is pumped by stretched second harmonic pulses of the Ti:sapphire laser [2]. The resulting pulses are $1.1 \mathrm{ps}$ long and tunable from $490 \mathrm{~nm}$ to $740 \mathrm{~nm}$. The three CARS beams are overlapped in a BOXCARS geometry and the time delay $t_{D}$ of the probe pulse with respect to the excitation pulses is scanned by a motorized linear stage. The CARS signal is detected time and frequency resolved by a spectrometer and an array detector.

This is an Open Access article distributed under the terms of the Creative Commons Attribution License 2.0, which permits unrestricted use, distribution, and reproduction in any medium, provided the original work is properly cited. 
The spectrally dispersed anti-Stokes signal is proportional to the squared Fourier transform of the nonlinear polarization which results from the interaction of the probe field at frequency $\omega_{p r}$ with the coherently excited modes. In the limit of ultrashort excitation due to the femtosecond Stokes pulse the coherence of a homogeneously broadened vibrational mode $i$ can be treated as a damped oscillator with frequency $\omega_{i}$ and dephasing time $T_{2 i}$ excited at time zero. After the crosscorrelation nonresonant contributions to the nonlinear polarization can be neglected. Assuming a narrowband probe pulse with a spectral bandwidth $\sigma_{p r}^{2}=\kappa_{p r} 2 \ln 2 / \pi^{2}$ the CARS signal can then be written as:

$$
S_{C A R S}\left(\omega, t_{D}\right)=\left|\sum_{i} A_{i} \exp \left[-\frac{\left(\omega_{i}+\omega_{p r}-\omega\right)^{2}}{4\left(\kappa_{p r}+\kappa_{i n h, i}\right)}\right] \exp \left[-\frac{\kappa_{p r}}{\kappa_{p r}+\kappa_{i n h, i}}\left(\frac{t_{D}}{T_{2 i}}+\kappa_{i n h, i} t_{D}^{2}\right)\right]\right|^{2}
$$

$A_{i}$ is the complex amplitude of the polarization due to mode $i$. Inhomogeneous broadening is taken into account by a Gaussian distribution of vibrational energies with a bandwidth $\sigma_{i n h}^{2}=\kappa_{i n h} 2 \ln 2 / \pi^{2}[3]$. A vibrational transition with negligible inhomogeneous broadening $\left(\kappa_{i n h}=0\right)$ results in a mono exponential decay with a dephasing independent line width which resembles the spectrum of the probe pulse. If the spectrum contains closely lying transitions within the probe width the modes interfere with each other. The resulting beating pattern may help to interpret complex spectra since the beating period is a precise measure for the frequency difference of the involved modes. If inhomogeneous dephasing processes become significant the line width of a CARS band is the geometric sum of the inhomogeneous line width and the width of the probe pulse. In addition the time evolution is no longer exponentially but contains a Gaussian damping term which can cause rapid signal decay.

\section{Application to liquids with overlapping Raman bands}

The Raman spectrum of cyclohexane exhibits three strong Raman bands around $2900 \mathrm{~cm}^{-1}$ resulting from $\mathrm{CH}$-stretch vibrations. A selection of CARS spectra at different time delays is presented in Figure 1a). The Raman mode at $2857 \mathrm{~cm}^{-1}$ is well separated from the other two contributions. With increasing probe delay the nonresonant background disappears and the shape of the band stays then constant. Due to negligible inhomogeneous broadening the signal decays mono exponentially (Fig. $1 \mathrm{~b}$, upper panel). Fitting an exponential function to the data for delay times after the crosscorrelation

a)

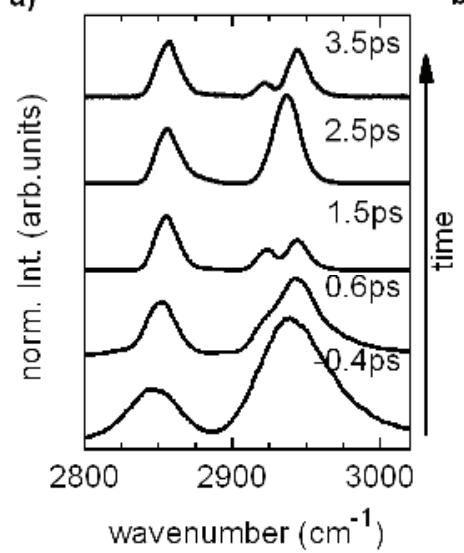

b)

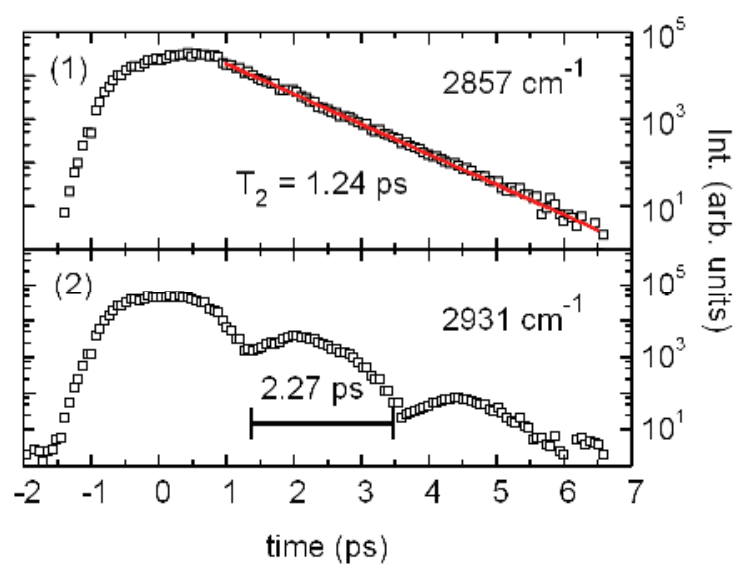

Fig. 1. a) CARS spectra of cyclohexane at different delay times. The spectra are normalized with respect to the signal at $2857 \mathrm{~cm}^{-1}$. b) Time traces of the signal at $2857 \mathrm{~cm}^{-1}$ and at $2930 \mathrm{~cm}^{-1}$. 
reveals a time constant of $0.62 \mathrm{ps}$ which reflects a dephasing time of $1.24 \mathrm{ps.} \mathrm{This} \mathrm{results} \mathrm{from} \mathrm{the}$ fact that the signal intensity scales with the square of the nonlinear polarization and decays therefore two times as fast.

The other two contributions around $2930 \mathrm{~cm}^{-1}$ behave quite differently and the temporal evolution of the two Raman bands is dominated by beating phenomena. The beating period of 2.27 ps can be extracted directly from time traces in the overlap region (Fig. 1b, lower panel). It corresponds to a frequency spacing of $14.7 \mathrm{~cm}^{-1}$ which matches exactly the difference of the two vibrational frequencies.

Fig. 2 shows the linear Raman and the CARS spectrum of the ionic liquid [C2mim][NTf2] at a time delay of $1.4 \mathrm{ps}$ in the spectral range of the alkyl $\mathrm{CH}$-stretch vibrations. Ionic liquids are promising solvents which are currently under intense investigation. The cation [C2mim] has a methyl and an ethyl side group. A broad and structured band results from several overlapping vibrational bands originating from different normal modes as well as due to the existence of different structural conformations. All contributions of the linear Raman measurement can also be seen in the CARS spectrum. However, the CARS spectrum is more structured and the signatures are more pronounced. The differences of the line positions are caused by interference effects. Destructive interference between neighboring modes enhances the contrast. A strong indication for this notion is the observation that the peak maxima originating from beating partners are shifted to opposite sides. Although the probe pulse is in this case not narrow enough to measure line widths smaller then the natural ones, an improved resolution is observed.

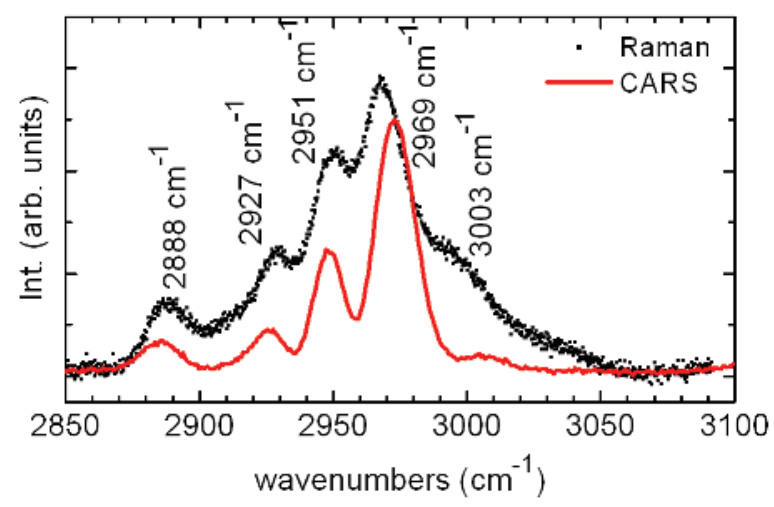

Fig. 2. Raman and CARS spectrum of the $\mathrm{CH}$ stretch vibrations of the alkyl chains of the cation in the ionic liquid [C2mim][NTf2].

In conclusion, the presented CARS technique is suitable to study dephasing processes and helps to disentangle homogenous and inhomogeneous contributions to the line width. Due to the increase of spectral resolution more details can be extracted in the case of overlapping bands. It is worth noting that the setup is also very suitable for pump-CARS probe experiments if it is combined with an infrared pump beam for investigating vibrational dynamics or an ultrashort ultraviolet excitation to study photoreactions. In these cases the short Stokes pulse defines the time resolution of the probing process which is here less than $50 \mathrm{fs}$.

\section{References}

1. W. Zinth, M. C. Nuss, W. Kaiser, Phys. Rev. A 30, 1139 (1984)

2. M. Lütgens, S. Chatzipapadopoulos, S. Lochbrunner, Opt. Express 20, 6478-6487 (2012)

3. R. F. Loring, S. Mukamel, J. Chem. Phys. 83, 2116 (1985) 